\title{
Light-triggered plasmonic vesicles with enhanced catalytic activity of glucose oxidase for programmable photothermal/starvation therapy
}

\author{
Zhuoting Deng ${ }^{\dagger}$, Yaling $\mathrm{He}^{\dagger}$, Muhammad Rizwan Younis, Shan Lei, Chao Jiang, Yanyan Yuan, \\ Peng Huang and Jing Lin
}

\begin{abstract}
Glucose oxidase (GOx)-based nanotheranostic agents hold great promise in tumor starvation and its synergistic therapy. Self-assembled plasmonic gold vesicles (GVs) with unique optical properties, large hollow cavity, and strong localized surface plasmon resonance, can be used as multi-functional nanocarriers for synergistic therapy. Herein, GOx-loaded GVs (GV-GOx) were developed for light-triggered GOx release as well as enhanced catalytic activity of GOx, achieving programmable photothermal/starvation therapy. Under near-infrared laser irradiation, the GV-GOx generated strong localized hyperthermia due to plasmon coupling effect of GVs, promoting the release of encapsulated GOx and increasing its catalytic activity, resulting in enhanced tumor starvation effect. In addition, the high photothermal effect improved the cellular uptake of GV-GOx and allowed an efficient monitoring of synergistic tumor treatment via photoacoustic/photothermal duplex imaging in vivo. Impressively, the synergistic photothermal/starvation therapy demonstrated complete tumor eradication in 4T1 tumorbearing mice, verifying superior synergistic anti-tumor therapeutic effects than monotherapy with no apparent systemic side effects. Our work demonstrated the development of a light-triggered nanoplatform for cancer synergistic therapy.
\end{abstract}

Keywords: light-triggered release, plasmonic vesicles, glucose oxidase, photothermal therapy, starvation therapy

\section{INTRODUCTION}

Tumor starvation therapy through chocking off the tumor site energy supply has attracted great interest in recent years [1-5]. Intratumoral glucose is one of the most common and essential energy supplies in tumor, which can be transformed to gluconic acid and hydrogen peroxide $\left(\mathrm{H}_{2} \mathrm{O}_{2}\right)$ through the catalysis of glucose oxidase (GOx) [6-9]. The GOx-mediated catalysis effectively consumes intratumoral glucose, which results in significant glucose depletion, thus triggering the tumor starvation process [10-14]. Although tumor starvation is an ideal tumor treatment approach, the poor reaction kinetics of enzyme-based catalysts and their safe delivery to the tumor site are the prominent bottlenecks, which impede its further applications [15].

Interestingly, it has been demonstrated that heat can accelerate the catalytic reactions, resulting in improved catalytic activity of catalysts [16-19]. However, the controlled generation of hyperthermia at the tumor site remains a hard nut to crack. As we know, photothermal therapy (PTT) involves photothermal agents to generate the localized hyperthermia under laser irradiation, resulting in ablation of tumor cells [20-23]. Integrating PTT with starvation therapy in one platform could realize the controlled generation of hyperthermia, which accelerates the catalytic reaction of GOx, achieving high antitumor effects due to the synergistic photothermally enhanced starvation therapy [24-26].

Additionally, the safe and controlled delivery of enzymes to avoid their potent toxicity and degradation remains a formidable challenge. In spite of the development of a wide range of nanocarriers such as liposomes [27], polymer micelles [28], polymersomes [29,30], and mesoporous silica [31], self-assembled plasmonic gold vesicles (GVs) [32-37] with near-infrared (NIR) absorption have aroused great research interest owing to their large hollow cavity and plasmonic coupling effect. These fea-

Marshall Laboratory of Biomedical Engineering, International Cancer Center, Laboratory of Evolutionary Theranostics (LET), School of Biomedical Engineering, Shenzhen University Health Science Center, Shenzhen 518060, China

${ }^{\dagger}$ These authors contributed equally to this work.

* Corresponding author (email: jingl@szu.edu.cn) 
tures enable GVs to (1) prevent the leakage of encapsulated therapeutic moiety (e.g., GOx), (2) laser-trigger the controllable moiety delivery, and (3) establish synergistic photothermally enhanced starvation therapy.

Herein, we developed GVs loaded with GOx (GV-GOx) to achieve light-triggered GOx release as well as enhanced catalytic activity of GOx for programmable photothermal/starvation therapy (Scheme 1). The GV-GOx was obtained by the self-assembly of gold nanoparticles (GNPs) grafted with a poly(ethylene glycol)- $b$-( $\varepsilon$-caprolactone) (PEO-b-PCL) block copolymer (BCP) through improved selective solvent approach. The as-prepared GV-GOx showed GOx loading capacity of $\sim 18 \mathrm{wt} \%$ and strong NIR absorption, which demonstrated excellent photoacoustic (PA) and photothermal duplex imaging. Under laser irradiation, GV-GOx generated strong localized hyperthermia, which triggered the controllable release of GOx and accelerated the GOx-mediated catalytic decomposition of glucose in tumor tissues simultaneously, resulting in significant glucose depletion for tumor starvation therapy. Collectively, by integrating PTT with starvation therapy, GV-GOx exhibited superior tumor inhibition efficacy in murine $4 \mathrm{~T} 1$ breast tumorbearing mice without any apparent systemic toxicity owing to the synergistic photothermally enhanced starvation therapy. These findings implied the biomedical potential of multifunctional plasmonic GV-based theranostic agent for cancer management.

\section{EXPERIMENTAL SECTION}

\section{Materials}

Chloroauric acid $\left(\mathrm{HAuCl}_{4} \cdot 3 \mathrm{H}_{2} \mathrm{O}\right)$, sodium citrate and GOx were purchased from J\&K. All solvents were obtained from Sigma-Aldrich unless indicated and used as received without any further purification. Deionized water (Millipore Milli-Q grade) with a resistivity of $18.2 \mathrm{M} \Omega \mathrm{cm}$ was used throughout this study.

\section{Preparation of GV-GOx}

GNPs $(26 \mathrm{~nm}$ ) were synthesized by the reduction of $\mathrm{HAuCl}_{4}$ using sodium citrate in aqueous phase. GV-GOx was prepared by assembling GNPs tethered with PEG- $b$ PCL copolymer using the improved selective solvent method. GNPs were modified with $\mathrm{PEO}_{45}-b-\mathrm{PCL}_{275}$ (number-averaged molecular weight $\left(M_{\mathrm{n}}\right)=$ $25.9 \mathrm{~kg} \mathrm{~mol}^{-1}$, polydispersity index $\left.(\mathrm{PDI})=1.43\right)$ in $N, N$ dimethylformamide (DMF). The polymer-modified GNPs in tetrahydrofuran (THF, $500 \mu \mathrm{L}, 1 \mathrm{mg} \mathrm{mL}^{-1}$ ) were slowly dripped into GOx aqueous solution $(500 \mu \mathrm{L}$, $1 \mathrm{mg} \mathrm{mL}^{-1}$ ) at a rate of $2 \mathrm{~mL} \mathrm{~h}^{-1}$ using an injection pump, then dialyzed in ultrapure water overnight. Finally, the

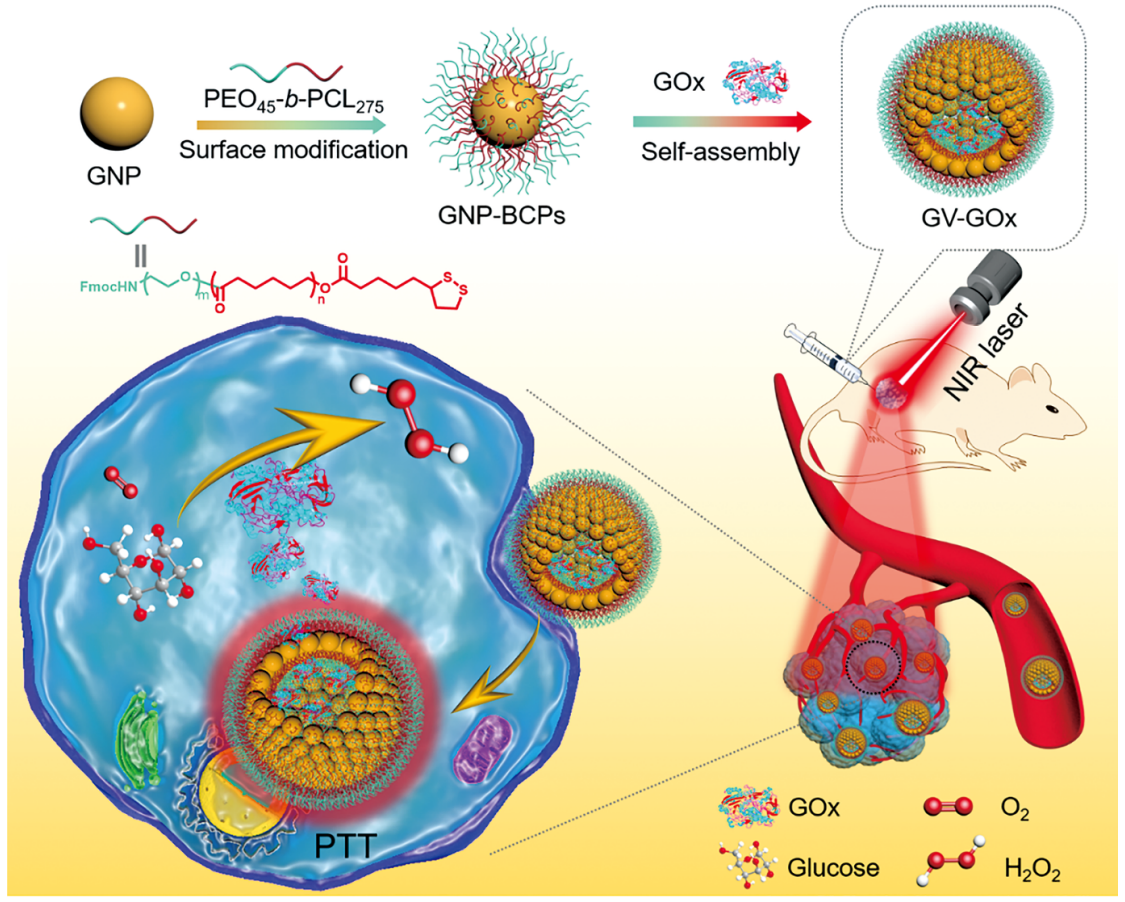

Scheme 1 Illustration of the fabrication of GV-GOx for programmable photothermal/starvation therapy. 
resulting solution was centrifuged and resuspended in aqueous solution for further studies.

\section{Characterizations of GV-GOx}

The morphologies of GV-GOx were characterized by FEIAPREO-S high-resolution scanning electron microscopy (SEM) and HITA CHI-HT7700 scanning transmission electron microscopy (TEM). Dynamic light scattering and zeta potential were recorded on Zeta sizer Nano-ZS90. Ultraviolet-visible (UV-Vis)-NIR absorption spectra were collected by Cary $60 \mathrm{UV}-\mathrm{Vis}$ spectrophotometer. Fourier transform infrared (FTIR) spectra were measured on a Spectrum Two Perkin Elmer spectrometer.

\section{GOx loading content}

The total amount of GOx and the amount of free GOx in the supernatant after dialysis were determined by protein quantification method [38]. GOx loading content was calculated by the formula: loading content $(\%)=($ total GOx - remaining content of GOx)/total GOx $\times 100 \%$.

\section{Evaluation of the activity of loaded GOx}

To estimate the GOx activity, GV-GOx (optical density $(\mathrm{OD}) @ 808 \mathrm{~nm}=0.4)$ solutions were mixed with different concentrations of glucose solutions: $20,15,5,4,2,1$, $0.5 \mathrm{mmol} \mathrm{L}^{-1}$, followed by laser irradiation $(808 \mathrm{~nm}$, $\left.1 \mathrm{~W} \mathrm{~cm}^{-2}, 10 \mathrm{~min}\right)$. Then $\mathrm{H}_{2} \mathrm{O}_{2}$ kit was used to detect the activity of $\mathrm{GOx}$, and the $\mathrm{pH}$ value was measured by using a $\mathrm{pH}$ meter. The solution of GV-GOx without laser irradiation was used as a control. Similarly, time-dependent generation of $\mathrm{H}_{2} \mathrm{O}_{2}$ and decrease of $\mathrm{pH}$ with/without laser irradiation were also monitored at a fixed concentration of glucose (20 and $3.2 \mathrm{mmol} \mathrm{L}^{-1}$, respectively).

\section{In vitro $\mathrm{PA}$ imaging}

Aqueous suspensions of GV-GOx with different optical densities (OD@808=0.1, 0.2,0.3,0.4,0.5) were prepared and their PA signals were recorded by a Vevo 2100 LAZR system. Ultrapure water was used as a control.

\section{In vitro cellular experiments}

4T1 cells were seeded into 96-well plates at a density of $5 \times 10^{3}$ cells/well $(n=5)$ and incubated at $37^{\circ} \mathrm{C}$ in DMEM media with $10 \%$ fetal bovine serum (FBS) $(100 \mu \mathrm{L})$ under $\mathrm{CO}_{2}$ atmosphere for $24 \mathrm{~h}$. For in vitro studies, all the irradiated groups were exposed to $808 \mathrm{~nm}$ laser at a power density of $1 \mathrm{~W} \mathrm{~cm}{ }^{-2}$ for $5 \mathrm{~min}$. For cytotoxicity assays, the cells were incubated with different concentrations of GVs or GV-GOx (equiv GOx: 0, 1.875, $3.75,7.5,15$ and $30 \mu \mathrm{g} \mathrm{mL}^{-1}$ ) for $12 \mathrm{~h}$. Cell viability was quantified by the methyl thiazolyl tetrazolium (MTT) assay.

In cell fluorescence imaging studies, the cells were incubated with GVs $\left(42.3 \mu \mathrm{g} \mathrm{mL}^{-1}\right)$ or GV-GOx $\left(42.3 \mu \mathrm{g} \mathrm{mL}^{-1}\right)$ for $4 \mathrm{~h}$, followed by treatment with/without laser irradiation. For live/dead cell observations, calcein acetoxymethyl ester (Calcein AM, live, green) and propidium iodide (PI, dead, red) dyes were added to each well, and then washed with serum-free DMEM. For intracellular reactive oxygen species (ROS) level observations, the cells were stained with $2^{\prime}, 7^{\prime}$-dichlorofluorescein diacetate (DCFH-DA) and washed. Finally, the images were acquired by a Nikon Eclipse Ts2 inverted fluorescence microscope. For subcellular localization, the cells were fixed, washed and then stained with DAPI for $10 \mathrm{~min}$, followed by washing again. Finally, images were acquired by a laser scanning confocal microscope.

\section{In vivo $\mathrm{PA} / \mathrm{photothermal} \mathrm{imaging}$}

Female BALB/c mice (4-5 weeks age) were purchased from Guangdong Medicinal Laboratory Animal Center and were used in accordance with the regulations of the Animal Ethical and Welfare Committee of Shenzhen University (AEWC-SZU). To build the subcutaneous xenograft model, 4T1 cells $\left(\sim 2 \times 10^{6}\right)$ were subcutaneously injected into the right flank of the mice $(18-22 \mathrm{~g})$. When the tumor size reached $\sim 60 \mathrm{~mm}^{3}, 50 \mu \mathrm{L}$ of phosphate-buffered saline (PBS), GVs $\left(400 \mu \mathrm{g} \mathrm{mL}^{-1}\right)$, or GV-GOx $\left(400 \mu \mathrm{g} \mathrm{mL}^{-1}\right)$ was injected into the tumorbearing mice. Then the tumors were exposed to $808 \mathrm{~nm}$ laser $\left(1 \mathrm{~W} \mathrm{~cm}^{-2}, 10 \mathrm{~min}\right)$. Thermal imaging was recorded by a SC300 infrared camera (FLIR). PA imaging and blood oxygen saturation were performed by a Vevo 2100 LAZR system.

\section{In vivo antitumor efficacy}

The mice were randomly divided into seven groups $(n=5$ per group), including (1) PBS $(50 \mu \mathrm{L})$, (2) PBS-Laser $(50 \mu \mathrm{L}), \quad(3) \quad \mathrm{GOx} \quad\left(283.7 \mu \mathrm{g} \mathrm{mL}^{-1}, 50 \mu \mathrm{L}\right)$, (4) GVs $\left(400 \mu \mathrm{g} \mathrm{mL}^{-1}, \quad 50 \mu \mathrm{L}\right), \quad(5) \mathrm{GVs}-\mathrm{Laser} \quad\left(400 \mu \mathrm{g} \mathrm{mL}^{-1}\right.$, $50 \mu \mathrm{L}),(6) \mathrm{GV}-\mathrm{GOx}\left(400 \mu \mathrm{gL}^{-1}, 50 \mu \mathrm{L}\right)$ and (7) GVGOx-Laser (GV-GOx-L, $\left.400 \mu \mathrm{gLL}^{-1}, 50 \mu \mathrm{L}\right)$. For irradiation groups, the tumors were exposed to $808 \mathrm{~nm}$ laser at a power density of $0.5 \mathrm{~W} \mathrm{~cm}^{-2}$ for $10 \mathrm{~min}$. The spot of laser beam was adjusted to cover the entire region of tumor. All the mice received once intratumoral injection on the first day. The size of the tumor was measured by a caliper every other day, and the tumor volume was calculated according to the formula: volume $=$ (length $\times$ width $\left.^{2}\right) / 2$. Besides, the body weights were also recorded 
during the treatment. All the mice were sacrificed after 15 days of treatment, and then the tumors and major organs were collected.

\section{Statistical analysis}

All data were expressed as means \pm standard deviation (SD). All figures shown in this article were obtained from at least three independent experiments with similar results. Statistical analyses between multiple groups were performed with the one-way ANOVA followed by Tukey's post-test or two-way ANOVA analysis followed by Bonferroni post-test (GraphPad Prism 6.0). Statistical significance is denoted by ${ }^{*} p<0.05,{ }^{* *} p<0.01$ and ${ }^{* * *} p$ $<0.001$.

\section{RESULTS AND DISCUSSION}

PEG- $b$-PCL copolymer was synthesized according to the reported protocol (Fig. S1a, b) [38]. GV-GOx was prepared by the improved selective solvent approach to maintain the catalytic activity of GOx. Briefly, the polymer-modified GNPs were slowly dripped into GOx aqueous solution to mix evenly, and then self-assembled by dialysis. The as-prepared GV-GOx exhibited the spherical morphology with high dispersity (Fig. 1a, b). The hydrodynamic size of GV-GOx is $\sim 245 \mathrm{~nm}$ with a polydispersity index of 0.19 (Fig. S2a). The surface zeta potential changes from -18 to $-29 \mathrm{mV}$ due to the loading of an anionic GOx into the cavity of GVs (Fig. S2b). The GV-GOx was homogenously dispersed in different physiological media including deionized water, PBS buffer, saline and cell culture medium containing 10\% FBS for over $24 \mathrm{~h}$ (Fig. S3). As shown in Fig. 1c, the UV-Vis-NIR absorption spectrum of GV-GOx showed a prominent red-shifted broad band from visible to NIR region after
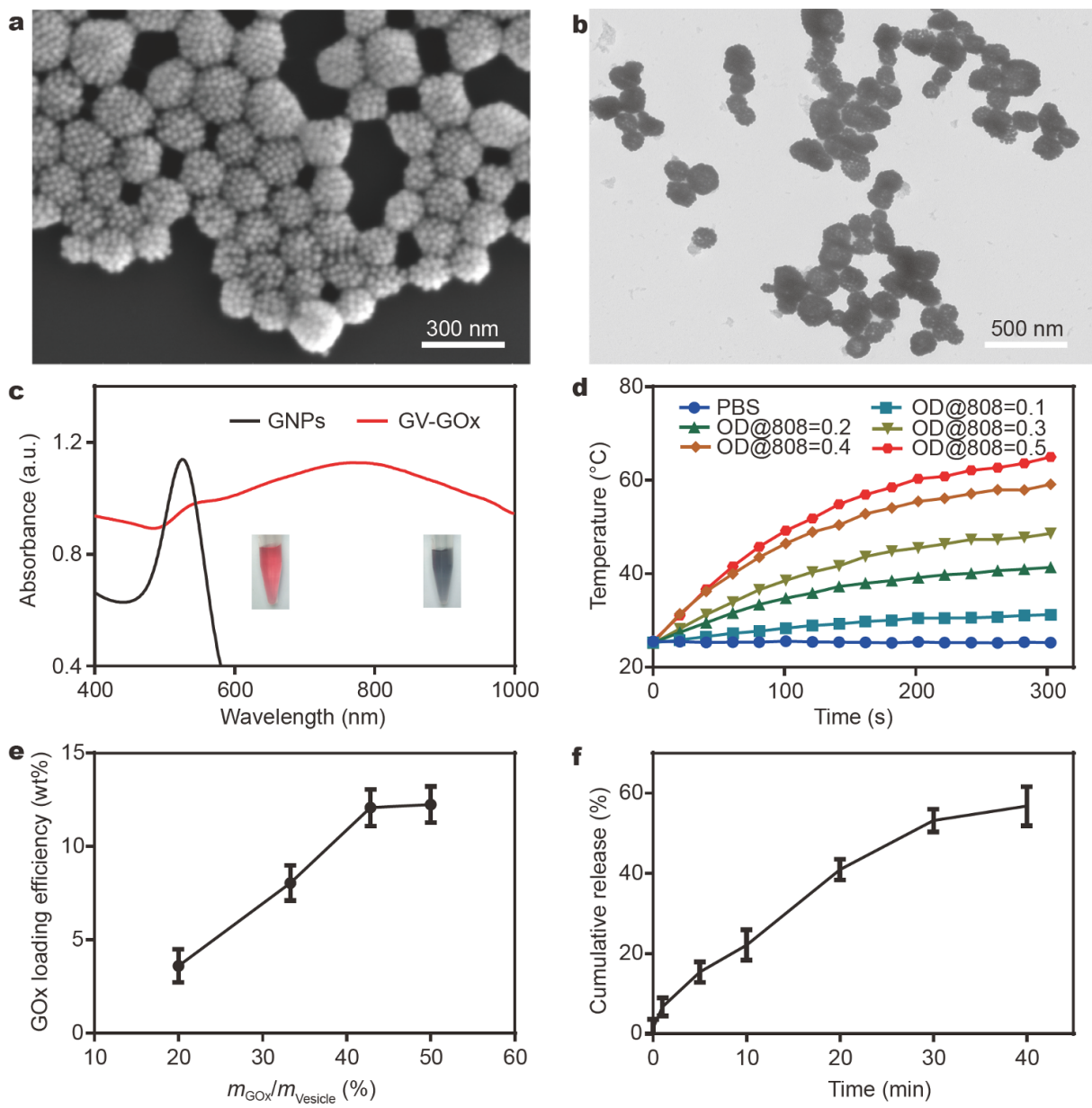

Figure 1 (a) SEM and (b) TEM images of GV-GOx self-assembled from GNPs. (c) UV-Vis-NIR spectra of GNPs and GV-GOx. (d) Temperature increase profile of varying concentrations of GV-GOx under laser irradiation. (e) GOx loading efficiency in GV-GOx as a function of GOx concentration. (f) GOx release profile at different times under laser irradiation. 
self-assembly, suggesting that GV-GOx could convert optical energy into heat. To investigate the light-to-heat conversion efficiency, varying concentrations of GV-GOx (OD@808=0.1,0.2, 0.3,0.4, and 0.5) underwent $808 \mathrm{~nm}$ laser irradiation at a power density of $1.0 \mathrm{~W} \mathrm{~cm}^{-2}$ for $5 \mathrm{~min}$ (Fig. 1d). An obvious concentration-dependent temperature increase was observed, whereas, by adjusting the content of GV-GOx, the temperature was precisely controlled from 25 to $63^{\circ} \mathrm{C}$, suggesting that GV-GOx possessed the capability of rapid and efficient conversion of NIR laser to generate strong localized hyperthermia. Then, the loading and light-regulated release of GOx in GV-GOx was determined. The GOx loading efficiency can reach to $\sim 12 \mathrm{wt} \%$ (Fig. 1e). To track the light-controlled GOx release of GV-GOx, GOx was conjugated with fluorescein isothiocyanate isomer (FITC) to afford GOx-FITC, verified by characteristic fluorescence peak (Fig. S4a) and FTIR spectra (Fig. S4b). The GOx-FITCloaded GVs have no fluorescence attributed to the fluorescence quenching by plasmonic GVs. Whereas, under laser irradiation $\left(808 \mathrm{~nm}, 1.0 \mathrm{~W} \mathrm{~cm}^{-2}\right)$, the enhanced fluorescence intensity of FITC was observed over time (Fig. S4c, d). The localized hyperthermia-triggered release of GOx reached nearly 55\% within $40 \mathrm{~min}$ (Fig. 1f). Meanwhile, morphological changes of GV-GOx further revealed that GV-GOx gradually disintegrated under irradiation (Fig. S5), facilitating the release of GOx. Meanwhile, in vitro PA imaging of GV-GOx was examined. As shown in Fig. S6a, GV-GOx showed strong PA signal, which was linearly correlated within a certain concentration range (Fig. S6b), allowing an effective treatment monitoring in vivo by $\mathrm{PA}$ imaging.

The catalytic activity of released GOx from the disintegrated GV-GOx was tested by determining the catalytic products $\left(\mathrm{H}_{2} \mathrm{O}_{2}\right.$ and gluconic acid) of $\mathrm{GOx}$ reaction (Fig. 2a). The generated $\mathrm{H}_{2} \mathrm{O}_{2}$ concentration of free GOxmediated catalytic decomposition of glucose was tested at different temperatures $\left(30,40,50\right.$ and $\left.60^{\circ} \mathrm{C}\right)$. As shown in Fig. S7, the catalytic activity of GOx can be maintained at $30-60^{\circ} \mathrm{C}$. Interestingly, the catalytic activity of GOx can be improved at high temperatures $\left(50\right.$ and $\left.60^{\circ} \mathrm{C}\right)$, which suggested mild hyperthermia could accelerate the catalytic reaction of GOx. Compared with GV-GOx, laserirradiated GV-GOx $\left(808 \mathrm{~nm}, 1.0 \mathrm{~W} \mathrm{~cm}^{-2}\right)$ generated significantly higher amount of $\mathrm{H}_{2} \mathrm{O}_{2}$, which is directly proportional to the glucose concentration (Fig. 2b) and time (Fig. 2c). The catalytic reaction of GOx and the produced gluconic acid lead to lower $\mathrm{pH}$. As shown in Fig. 2d and e, a rapid decrease in the $\mathrm{pH}$ value was recorded with glucose concentration and time upon laser irradiation. These results strongly dictate the light-triggered release as well as enhanced catalytic activity of GOx.

Next, in vitro cytotoxicity of GV-GOx was evaluated by MTT assay on HEK293T and 4T1 cells, respectively. As shown in Fig. 3a, the cell viabilities were over $80 \%$ at all tested concentrations $\left(0-30 \mu \mathrm{g} \mathrm{mL}^{-1}\right)$, which suggests GV-GOx is safe under these concentrations. The viability of free GOx-treated 4T1 cells was less than $20 \%$ when GOx concentration was over $1.6 \mu \mathrm{g} \mathrm{mL}^{-1}$ (Fig. S8), which suggests the GV carrier can dramatically reduce the toxicity of GOx. Subsequently, the in vitro programmable photothermal/starvation therapy was evaluated on 4T1 cells. Neither the GVs nor GV-GOx decreased the cellular viability in dark. In contrast, under laser irradiation (1.0 $\mathrm{W} \mathrm{cm}^{-2}, 5 \mathrm{~min}$ ), GV-GOx exhibited more significant cellular destruction of $4 \mathrm{~T} 1$ cells than all other groups, which was attributed to the synergistic effect of photothermal/starvation therapy (Fig. 3b).

In addition, live/dead assay using Calcein AM/PI costaining images further endorsed the higher synergistic therapeutic effects than single therapy (Fig. S9). To ensure that the higher killing effect is caused by the synergistic effects, we verified the catalytic activity of GOx at the cellular level using DCFH-DA, an ROS fluorescence probe. Compared with other groups, a markedly higher fluorescence intensity of DCFH-DA was observed in the cells treated with GV-GOx under laser irradiation (Fig. 3c), which confirmed the generation of large amount of intracellular ROS $\left(\mathrm{H}_{2} \mathrm{O}_{2}\right)$ by GV-GOx owing to the photothermally enhanced catalytic activity of GOx. Irradiation-enhanced cellular uptake of GV-GOx was also investigated. As shown in Fig. 3d, a higher fluorescence intensity was observed from NIR laser-treated cells incubated with FITC-labeled GV-GOx, suggesting lighttriggered cellular uptake enhancement.

Because of high NIR absorption and good photothermal effect in vitro, GV-GOx was anticipated to be a high-performance photothermal/PA imaging agent. As shown in Fig. 4a and b, both the GVs and GV-GOx increased the local tumor temperature up to $47^{\circ} \mathrm{C}$ under laser irradiation $\left(808 \mathrm{~nm}, 0.5 \mathrm{~W} \mathrm{~cm}^{-2}, 10 \mathrm{~min}\right)$, which implies that the GV-GOx can act as a photothermal agent to mediate efficient photothermal destruction of tumor cells in vivo. In addition, PA imaging was also conducted to evaluate the PA properties of GV-GOx. Strong PA signals were observed in the tumor region (Fig. 4c), which was approximately 10 -fold stronger than pre-injection (Fig. 4d). Three-dimensional (3D) PA imaging of tumor tissue also showed a strong contrast before and after GVGOx injection (Fig. 4e). Given the fact that the GOx- 
a
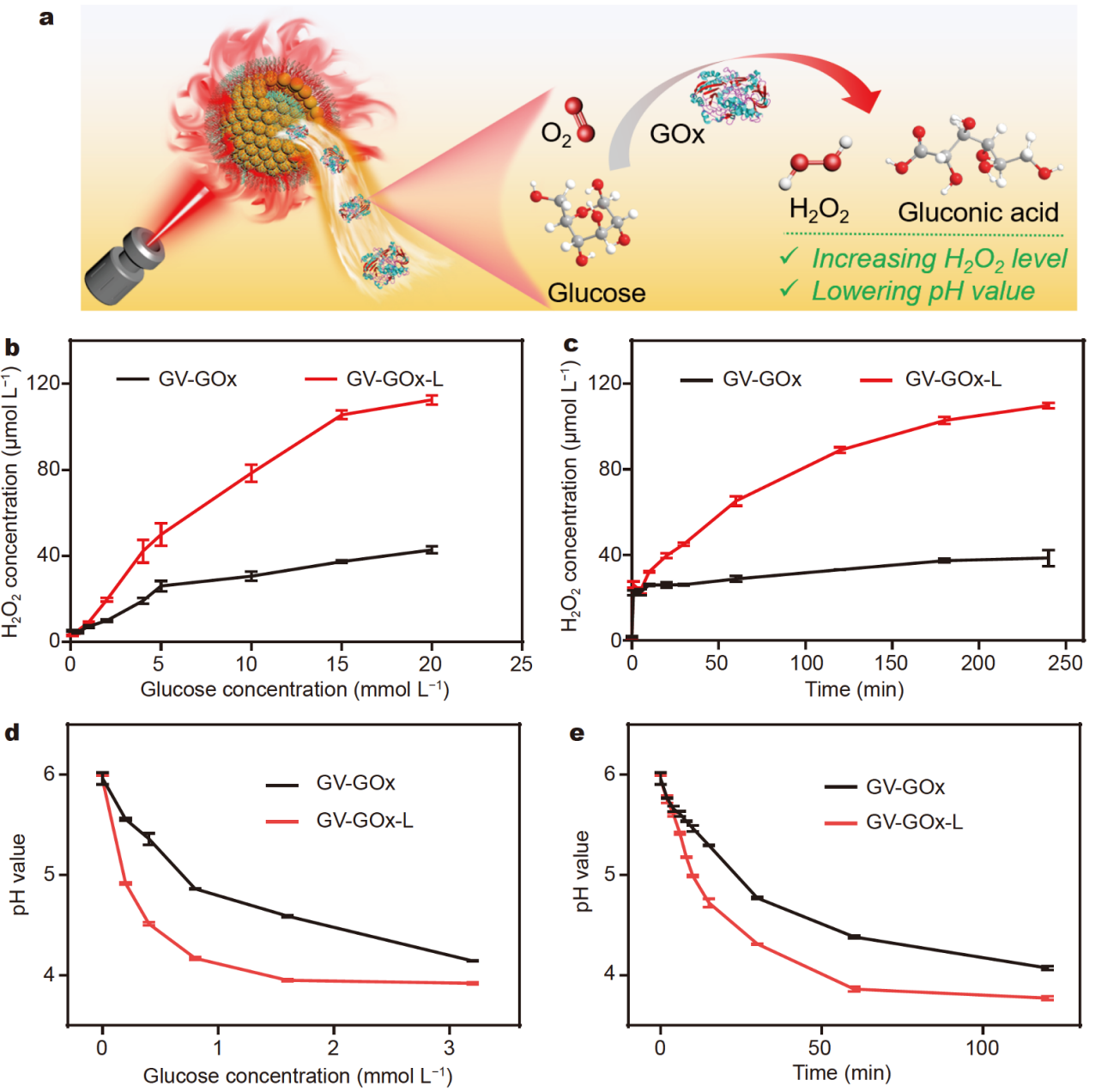

Figure 2 (a) Illustration of light-triggered GOx catalysis reaction. (b) Glucose-concentration-dependent and (c) time-dependent $\mathrm{H}_{2} \mathrm{O}_{2}$ generation of GV-GOx with/without laser irradiation. (d) Glucose-concentration-dependent and (e) time-dependent decrease in pH value of GV-GOx with/without laser irradiation.

mediated catalytic decomposition of glucose consumed a large amount of endogenous oxygen $\left(\mathrm{O}_{2}\right)$, the intratumoral blood oxygen saturation $\left(\mathrm{sO}_{2}\right)$ concentration significantly dropped to $10 \%$ after laser irradiation of GVGOx for $10 \mathrm{~min}$, while the mice in the GVs group maintained their original $\mathrm{O}_{2}$ levels (Fig. $4 \mathrm{f}$ and Fig. S10) in accordance with $3 \mathrm{D}$ images of $\mathrm{sO}_{2}$ (Fig. S11). These results suggest the light-triggered release and enhanced catalytic activity of GOx in the tumor tissues lead to the $\mathrm{O}_{2}$ consumption during the catalytic reaction of GOx.

Finally, the in vivo antitumor efficacy of GV-GOx was evaluated on $4 \mathrm{~T} 1$ tumor-bearing mice. As shown in Fig. 5a, the complete tumor elimination in GV-GOx-L group was observed after 15 day of treatment, whereas, negligible tumor growth inhibition was seen in other control groups, suggesting the prominent antitumor ef- fect of GV-GOx-L. Meanwhile, no body weight loss was observed during the treatment (Fig. 5b), indicating that GV-GOx has good biocompatibility. To quantitatively assess the antitumor efficacy, the tumor weights were measured and recorded (Fig. 5c). In the absence or presence of laser irradiation, the PBS group had more than 12 -fold increase of tumor weight, indicating that laser irradiation alone is unable to trigger the anticancer effect. Without laser irradiation, the tumors in GVs-treated mice grew to the same size as those in PBS or GOx-treated mice, while GVs-L exhibited much slower tumor growth than control groups, suggesting a certain level of anticancer effects due to the photothermal effect. Impressively, GV-GOx-L demonstrated almost complete tumor growth inhibition throughout the entire experimental period with a tumor inhibition rate of nearly $100 \%$. The photographs 

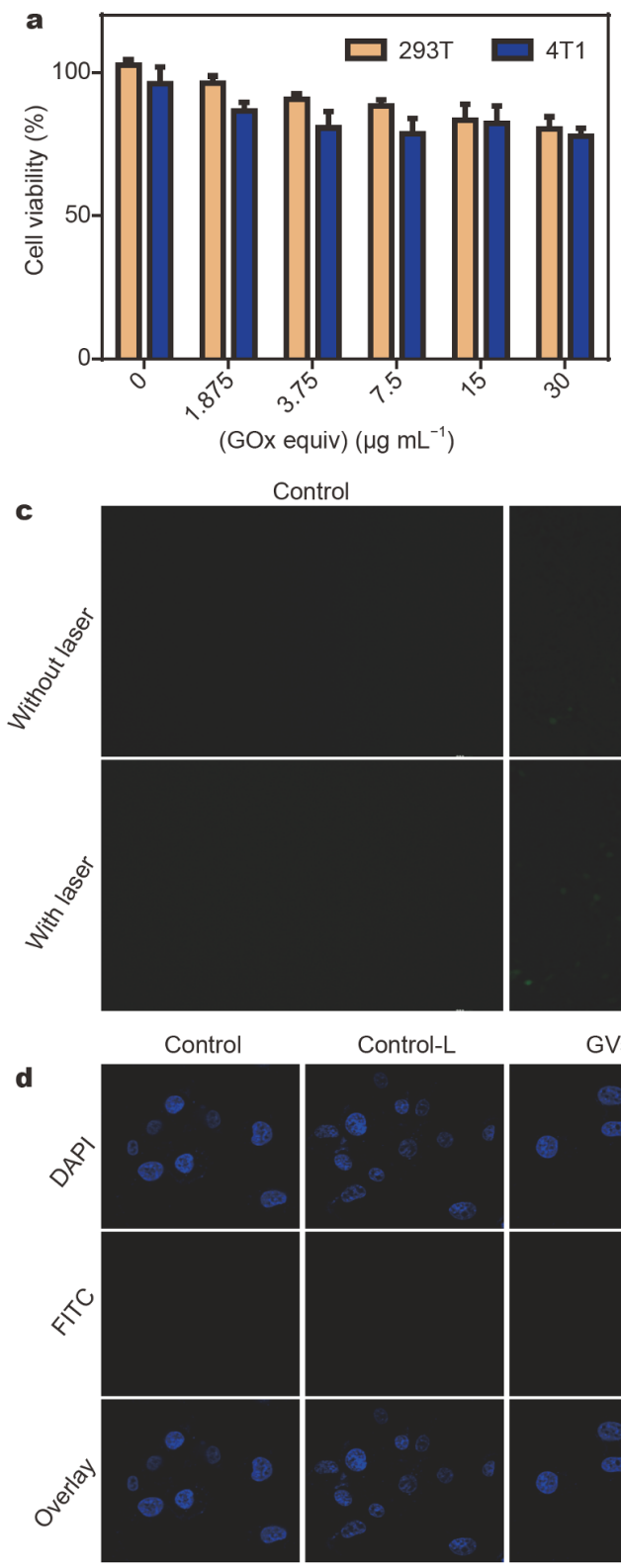

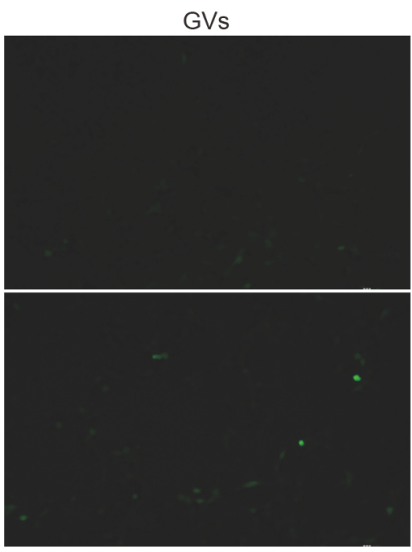

GVs
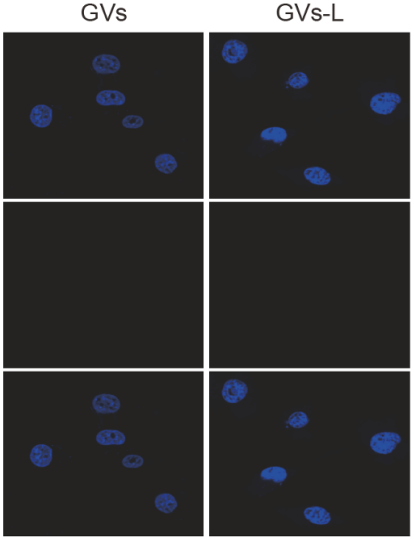

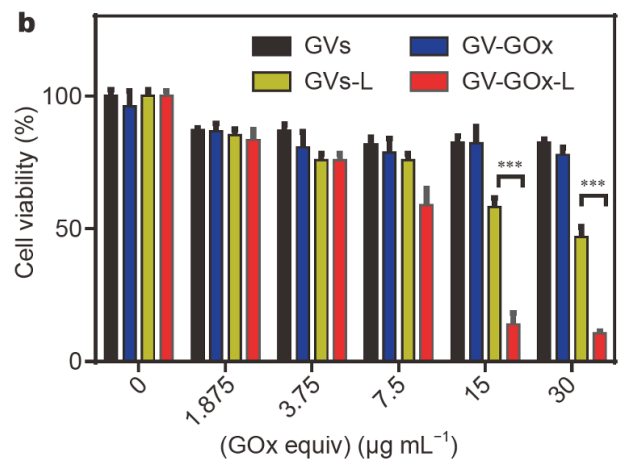

GV-GOX

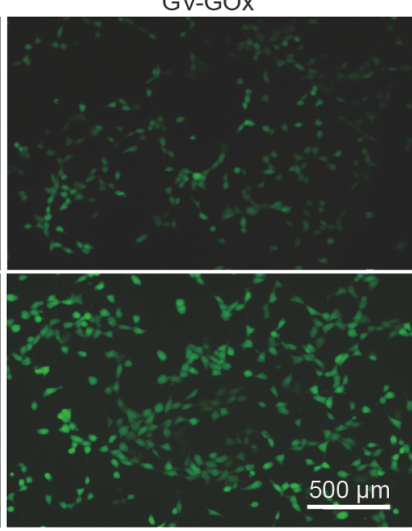

GV-GOX

GV-GOX-L

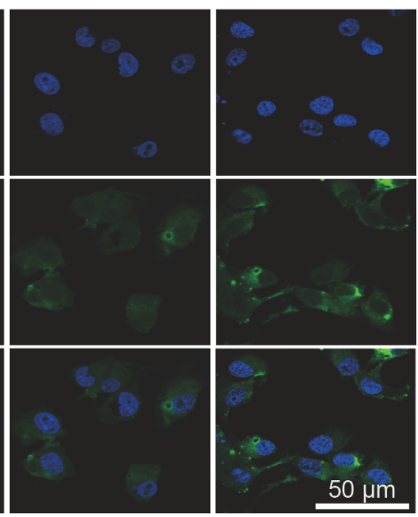

Figure 3 (a) Dark cytotoxicity of GV-GOx in 4T1 and HEK 293T cells. (b) In vitro therapeutic efficacy after incubated with varying concentrations of GVs or GV-GOx with/without laser irradiation. ${ }^{* * *} p<0.001$. (c) Fluorescence images of DCFH-DA (scale bar $\left.=500 \mu \mathrm{m}\right)$ and (d) confocal fluorescence images (scale bar $=50 \mu \mathrm{m}$ ) in $4 \mathrm{~T} 1$ cells incubated with GVs or GV-GOx with/without laser irradiation.

of $4 \mathrm{~T} 1$ tumor-bearing mice at the 15 day further confirmed that the tumors in the GV-GOx-L group were completely ablated (Fig. 5d). H\&E stained images further showed that GV-GOx-L significantly damaged the tumor cells (Fig. 5e), while caused negligible damage/ inflammation to other organs (Fig. S12). All results indicate that the light simultaneously triggered GOx release and enhanced GOx activity, achieving synergetic photothermal/starvation therapy and leading to nearly complete tumor destruction without any tumor recurrence.

The delivery of GOx by GVs shows the following advantages: (1) high GOx loading efficiency ( 12 wt\%) due to the hollow interior of GVs; (2) the controllable release of GOx by laser irradiation; (3) enhanced catalytic activity of GOx by laser irradiation; (4) PA/photothermal duplex imaging-guided synergistic photothermal/starvation therapy with improved treatment efficacy. In addition, 

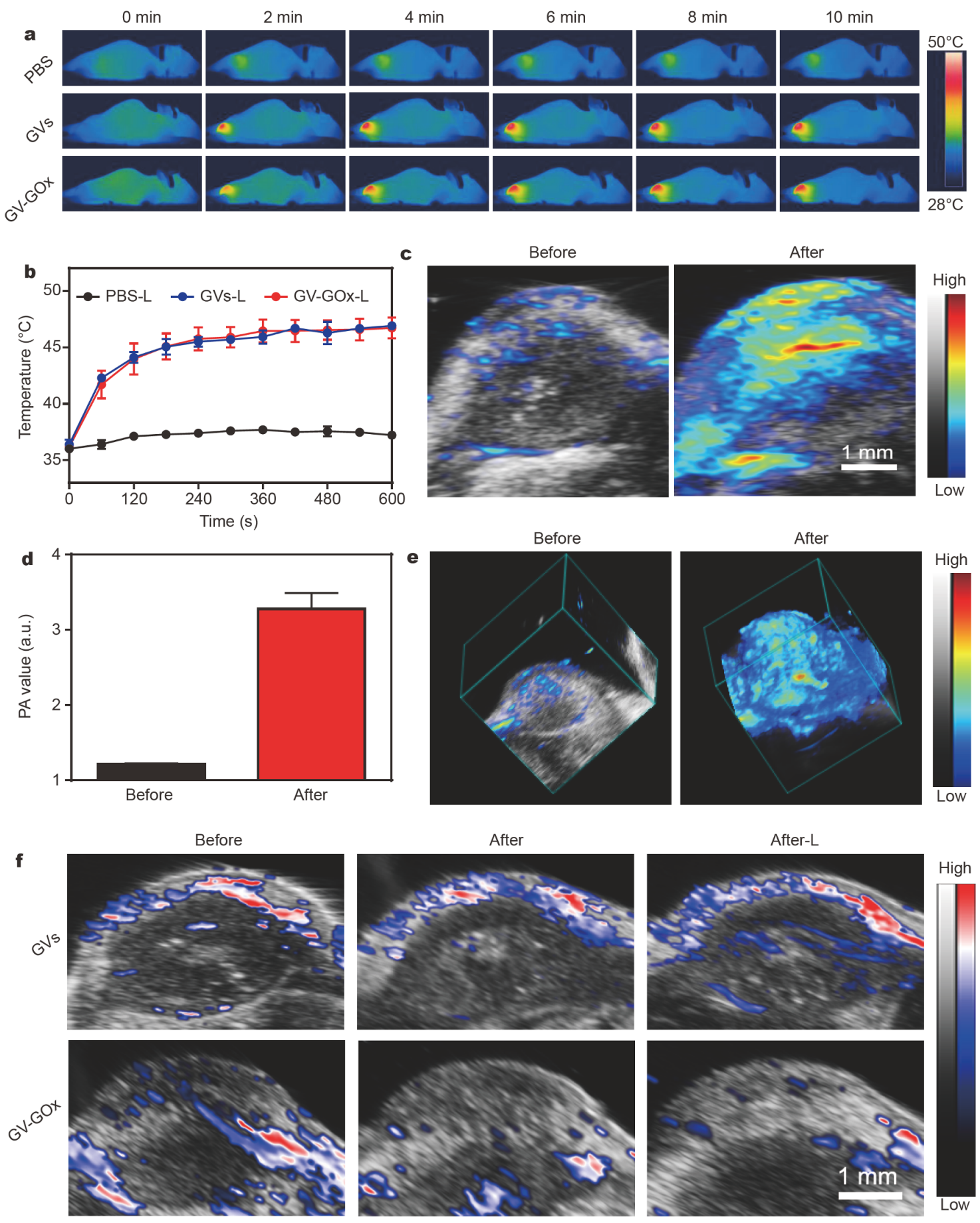

Figure 4 (a) Thermal images in the tumor region treated with PBS, GVs and GV-GOx under laser irradiation. (b) The corresponding temperatures at different time points of (a). (c) In vivo PA images (scale bar $=1 \mathrm{~mm}$ ), (d) average PA intensity and (e) 3D PA images of tumor tissues before and after injection of GV-GOx. (f) In vivo oxygen saturation $\left(\mathrm{sO}_{2}\right)$ images of tumor tissues before/after injection and after laser irradiation $(\mathrm{scale}$ bar $=1 \mathrm{~mm})$.

the multifunctional nanoplatform has high solubility and stability in aqueous solution and excellent biocompatibility, thus promising for biomedical applications.

\section{CONCLUSIONS}

In summary, GOx-loaded GVs have been explored as a nanotheranostic agent for duplex imaging-guided sy- 

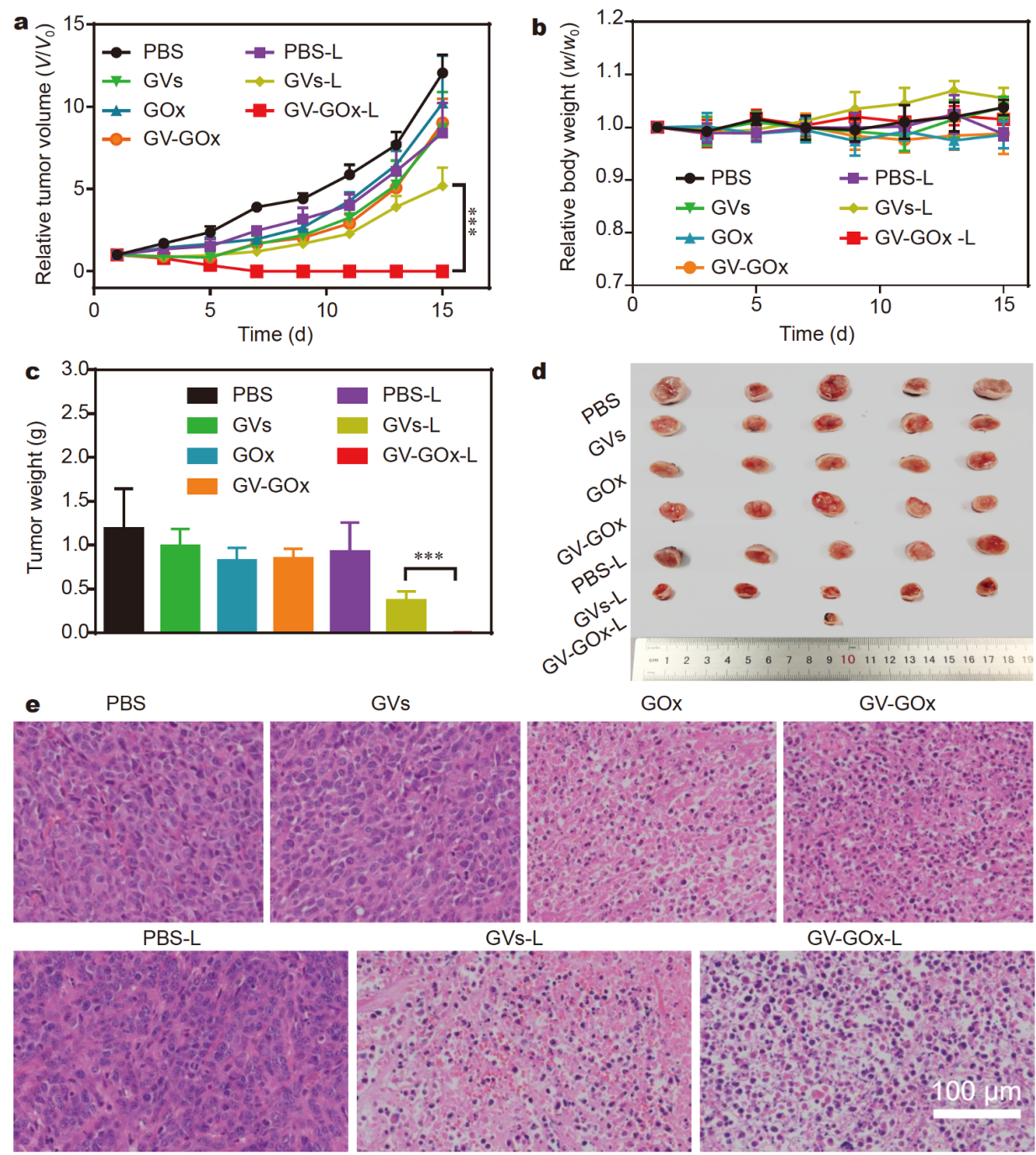

Figure 5 (a) Time-dependent tumor growth curves, (b) relative body weight and (c) tumor weight from 4T1-tumor bearing mice with different treatments $(n=5$, mean \pm SD). (d) Photographs and (e) H\&E stained tumor sections at the Day 15 after different treatments $($ scale bar $=100 \mu \mathrm{m})$.

nergistic photothermal/starvation cancer therapy. Due to the plasmon coupling effect, the GV-GOx exhibited strong photothermal effect, generating strong localized hyperthermia to trigger the release of encapsulated GOx as well as enhance its catalytic activity, resulting in photothermally augmented tumor starvation to realize complete $4 \mathrm{~T} 1$ tumor eradication in vivo. Our study demonstrated GVs, as a multifunctional cancer nanotheranostic platform with high antitumor efficacy and biological safety, hold biomedical potential for cancer management.

Received 22 June 2020; accepted 24 August 2020; published online 17 November 2020

1 Zhang C, Ni D, Liu Y, et al. Magnesium silicide nanoparticles as a deoxygenation agent for cancer starvation therapy. Nat Nanotech, 2017, 12: 378-386

2 Gao F, Tang Y, Liu WL, et al. Intra/extracellular lactic acid exhaustion for synergistic metabolic therapy and immunotherapy of tumors. Adv Mater, 2019, 31: 1904639

3 Yu S, Chen Z, Zeng X, et al. Advances in nanomedicine for cancer starvation therapy. Theranostics, 2019, 9: 8026-8047

4 Zhang $\mathrm{Y}$, Wan $\mathrm{Y}$, Liao $\mathrm{Y}$, et al. Janus $\gamma-\mathrm{Fe}_{2} \mathrm{O}_{3} / \mathrm{SiO}_{2}$-based nanotheranostics for dual-modal imaging and enhanced synergistic cancer starvation/chemodynamic therapy. Sci Bull, 2020, 65: 564572

5 Zeng L, Huang K, Wan Y, et al. Programmable starving-photodynamic synergistic cancer therapy. Sci China Mater, 2020, 63: 611-619

6 Wang M, Wang D, Chen Q, et al. Recent advances in glucoseoxidase-based nanocomposites for tumor therapy. Small, 2019, 15: 1903895

7 Fu LH, Qi C, Hu YR, et al. Glucose oxidase-instructed multimodal 
synergistic cancer therapy. Adv Mater, 2019, 31: 1808325

8 Fu LH, Hu YR, Qi C, et al. Biodegradable manganese-doped calcium phosphate nanotheranostics for traceable cascade reactionenhanced anti-tumor therapy. ACS Nano, 2019, 13: 1398513994

9 Yu Z, Zhou P, Pan W, et al. A biomimetic nanoreactor for synergistic chemiexcited photodynamic therapy and starvation therapy against tumor metastasis. Nat Commun, 2018, 9: 5044

10 Fan W, Lu N, Huang P, et al. Glucose-responsive sequential generation of hydrogen peroxide and nitric oxide for synergistic cancer starving-like/gas therapy. Angew Chem Int Ed, 2017, 56: 1229-1233

11 Zhang L, Wan SS, Li CX, et al. An adenosine triphosphateresponsive autocatalytic Fenton nanoparticle for tumor ablation with self-supplied $\mathrm{H}_{2} \mathrm{O}_{2}$ and acceleration of $\mathrm{Fe}(\mathrm{III}) / \mathrm{Fe}(\mathrm{II})$ conversion. Nano Lett, 2018, 18: 7609-7618

12 Zhang L, Wang Z, Zhang Y, et al. Erythrocyte membrane cloaked metal-organic framework nanoparticle as biomimetic nanoreactor for starvation-activated colon cancer therapy. ACS Nano, 2018, 12: 10201-10211

13 Zhang Y, Yang Y, Jiang S, et al. Degradable silver-based nanoplatform for synergistic cancer starving-like/metal ion therapy. Mater Horiz, 2019, 6: 169-175

14 Ma Y, Zhao Y, Bejjanki NK, et al. Nanoclustered cascaded enzymes for targeted tumor starvation and deoxygenation-activated chemotherapy without systemic toxicity. ACS Nano, 2019, 13: 88908902

15 Fu LH, Qi C, Lin J, et al. Catalytic chemistry of glucose oxidase in cancer diagnosis and treatment. Chem Soc Rev, 2018, 47: 64546472

16 Tang Z, Liu Y, He M, et al. Chemodynamic therapy: Tumour microenvironment-mediated Fenton and Fenton-like reactions. Angew Chem Int Ed, 2019, 58: 946-956

17 Liang R, Li Y, Huo M, et al. Triggering sequential catalytic Fenton reaction on $2 \mathrm{D}$ Mxenes for hyperthermia-augmented synergistic nanocatalytic cancer therapy. ACS Appl Mater Interfaces, 2019, 11: 42917-42931

18 Feng W, Han X, Wang R, et al. Nanocatalysts-augmented and photothermal-enhanced tumor-specific sequential nanocatalytic therapy in both NIR-I and NIR-II biowindows. Adv Mater, 2019, 31: 1805919

19 Gao G, Jiang Y, Guo Y, et al. Enzyme-mediated tumor starvation and phototherapy enhance mild-temperature photothermal therapy. Adv Funct Mater, 2020, 30: 1909391

20 Younis MR, Wang C, An R, et al. Low power single laser activated synergistic cancer phototherapy using photosensitizer functionalized dual plasmonic photothermal nanoagents. ACS Nano, 2019, 13: acsnano.8b09552

21 Younis MR, An RB, Yin YC, et al. Plasmonic nanohybrid with high photothermal conversion efficiency for simultaneously effective antibacterial/anticancer photothermal therapy. ACS Appl Bio Mater, 2019, 2: 3942-3953

22 Chen Q, Xu L, Liang C, et al. Photothermal therapy with immuneadjuvant nanoparticles together with checkpoint blockade for effective cancer immunotherapy. Nat Commun, 2016, 7: 13193

23 Wang X, Zhong X, Zha Z, et al. Biodegradable $\mathrm{CoS}_{2}$ nanoclusters for photothermal-enhanced chemodynamic therapy. Appl Mater Today, 2020, 18: 100464

$24 \mathrm{Hu}$ JJ, Liu MD, Gao F, et al. Photo-controlled liquid metal nanoparticle-enzyme for starvation/photothermal therapy of tumor by win-win cooperation. Biomaterials, 2019, 217: 119303

25 Zhang T, Li Y, Hong W, et al. Glucose oxidase and polydopamine functionalized iron oxide nanoparticles: Combination of the photothermal effect and reactive oxygen species generation for dualmodality selective cancer therapy. J Mater Chem B, 2019, 7: 21902200

26 He $\mathrm{T}, \mathrm{Xu} \mathrm{H}$, Zhang $\mathrm{Y}$, et al. Glucose oxidase-instructed traceable self-oxygenation/hyperthermia dually enhanced cancer starvation therapy. Theranostics, 2020, 10: 1544-1554

27 Zhang R, Song X, Liang C, et al. Catalase-loaded cisplatin-prodrugconstructed liposomes to overcome tumor hypoxia for enhanced chemo-radiotherapy of cancer. Biomaterials, 2017, 138: 13-21

28 Miller MA, Askevold B, Mikula H, et al. Nano-palladium is a cellular catalyst for in vivo chemistry. Nat Commun, 2017, 8: 15906

29 Geilich BM, Gelfat I, Sridhar S, et al. Superparamagnetic iron oxide-encapsulating polymersome nanocarriers for biofilm eradication. Biomaterials, 2017, 119: 78-85

30 Nishimura T, Sasaki Y, Akiyoshi K. Biotransporting self-assembled nanofactories using polymer vesicles with molecular permeability for enzyme prodrug cancer therapy. Adv Mater, 2017, 29: 1702406

31 Huo M, Wang L, Chen Y, et al. Tumor-selective catalytic nanomedicine by nanocatalyst delivery. Nat Commun, 2017, 8: 357

32 Song J, Pu L, Zhou J, et al. Biodegradable theranostic plasmonic vesicles of amphiphilic gold nanorods. ACS Nano, 2013, 7: 9947 9960

33 Ye H, Yang K, Tao J, et al. An enzyme-free signal amplification technique for ultrasensitive colorimetric assay of disease biomarkers. ACS Nano, 2017, 11: 2052-2059

34 Huang Y, Huang P, Lin J. Plasmonic gold nanovesicles for biomedical applications. Small Methods, 2019, 3: 1800394

35 Lin J, Wang S, Huang P, et al. Photosensitizer-loaded gold vesicles with strong plasmonic coupling effect for imaging-guided photothermal/photodynamic therapy. ACS Nano, 2013, 7: 5320-5329

36 Song J, Yang X, Jacobson O, et al. Sequential drug release and enhanced photothermal and photoacoustic effect of hybrid reduced graphene oxide-loaded ultrasmall gold nanorod vesicles for cancer therapy. ACS Nano, 2015, 9: 9199-9209

37 Liu Y, He J, Yang K, et al. Folding up of gold nanoparticle strings into plasmonic vesicles for enhanced photoacoustic imaging. Angew Chem Int Ed, 2015, 54: 15809-15812

38 Jiang S, Zhang Y, Yang Y, et al. Glucose oxidase-instructed fluorescence amplification strategy for intracellular glucose detection. ACS Appl Mater Interfaces, 2019, 11: 10554-10558

Acknowledgements This work was supported by the National Natural Science Foundation of China (31771036 and 51703132), the Basic Research Program of Shenzhen (JCYJ20180507182413022 and JCYJ20170412111100742), Guangdong Province Natural Science Foundation of Major Basic Research and Cultivation Project (2018B030308003), and Fok Ying-Tong Education Foundation for Young Teachers in the Higher Education Institutions of China (161032). We thank the Instrumental Analysis Center of Shenzhen University (Lihu Campus).

Author contributions Lin J conceived the idea, supervised the study and wrote the manuscript; Deng $\mathrm{Z}$ participated in the fabrication and characterization of GV-GOx; Jiang C and Yuan Y participated in the photoacoustic imaging; Deng $\mathrm{Z}$ and $\mathrm{He} \mathrm{Y}$ completed the subsequent experiments, drew all figures and wrote this manuscript; Lin J, Huang P, Younis MR and Lei S helped editing this manuscript. 
Conflict of interest The authors declare that they have no conflict of interest.

Supplementary information online version of the paper.

Supporting data are available in the

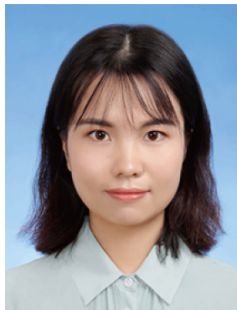

Zhuoting Deng received her BSc degree in pharmaceutical engineering from the Southeast University in 2017. Currently, she is studying for her MSc degree in the Laboratory of Evolutionary Theranostics (LET), School of Biomedical Engineering, Shenzhen University. Her current research interests mainly focus on the development of multifunctional nanotherapeutic agent based on gold nanovesicles and their applications in cancer therapy.

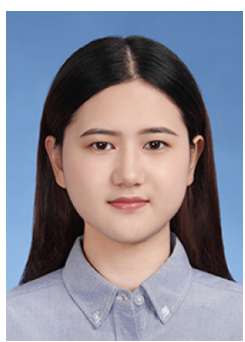

Yaling He received her BSc degree from Anhui Normal University in 2018. Currently, she is studying for her MSc degree in the LET, School of Biomedical Engineering, Shenzhen University. Her current research interests mainly focus on the development of $\mathrm{pH}$-sensitive gold vesicles for biomedical applications.

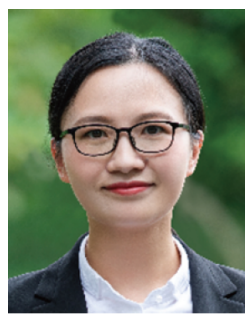

Jing Lin is a Distinguished Professor at the School of Biomedical Engineering, Shenzhen University Health Science Center, China. She received her $\mathrm{PhD}$ degree in organic chemistry from Donghua University and Shanghai Institute of Organic Chemistry, Chinese Academy of Sciences in 2010. Then she joined the PharmaResources (Shanghai) Co., Ltd. as a group leader. After two years, she moved to the United States of America and spent four years as a postdoc at the University of Maryland and the National Institutes of Health (NIH). She joined the faculty of Shenzhen University in 2016 and was promoted as a Distinguished Professor in 2018. Her research focuses on molecular imaging, nanomedicine and theranostics.
光触发增强葡萄糖氧化酶催化活性的等离子体囊 泡用于程序化光热-饥饿疗法

邓卓婷 ${ }^{\dagger}$, 何亚玲 ${ }^{\dagger}$, Muhammad Rizwan Younis, 雷珊, 蒋超, 袁燕燕, 黄鹏, 林静*

摘要 在肿瘤的饥饿治疗及协同治疗中，基于葡萄糖氧化酶(GOx) 的纳米诊疗剂展现出具大的应用前景. 自组装等离子体金囊泡 (GV), 由于具有独特的光学性能、巨大空腔和强局域表面等离子 体共振等特性, 可作为协同治疗的多功能纳米载体. 本文中, 我们 开发了一种装载GOx的GV(GV-GOx) 用于光触发释放GOx, 同时增 强 $\mathrm{GOx}$ 的催化活性, 从而实现程序化光热-饥饿治疗. 在近红外激光 照射下, 由于 GV具有等离子体耦合效应, GV-GOx可以产生很强的 局部高热, 引起封装的GOx释放, 同时高热可提高GOx催化活性, 从 而增强肿瘤的饥饿效应. 此外, 高光热效应可促进细胞对GV-GOx 的摄取, 并可通过活体光声/光热双模态成像对协同治疗进行有效 监测. 令人印象深刻的是, 协同光热/饥饿疗法能完全消融 $4 \mathrm{~T} 1$ 荷瘤 小鼠的肿瘤, 抗肿瘤效果明显优于单一疗法, 且没有明显的系统毒 性. 本工作展示了一种光触发的纳米平台, 可用于癌症协同治疗. 